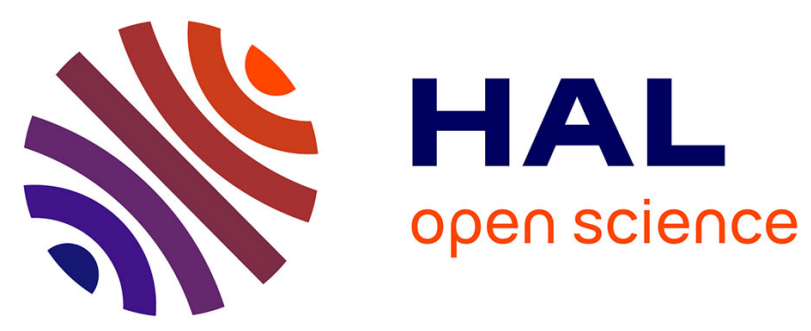

\title{
Primal-dual splitting algorithm for solving inclusions with mixtures of composite, Lipschitzian, and parallel-sum type monotone operators

\author{
Patrick Louis Combettes, Jean-Christophe Pesquet
}

\section{To cite this version:}

Patrick Louis Combettes, Jean-Christophe Pesquet. Primal-dual splitting algorithm for solving inclusions with mixtures of composite, Lipschitzian, and parallel-sum type monotone operators. Set-Valued and Variational Analysis, 2012, 20 (2), pp.307-330. 10.1007/s11228-011-0191-y · hal-00794044

\author{
HAL Id: hal-00794044 \\ https://hal.science/hal-00794044
}

Submitted on 25 Feb 2013

HAL is a multi-disciplinary open access archive for the deposit and dissemination of scientific research documents, whether they are published or not. The documents may come from teaching and research institutions in France or abroad, or from public or private research centers.
L'archive ouverte pluridisciplinaire HAL, est destinée au dépôt et à la diffusion de documents scientifiques de niveau recherche, publiés ou non, émanant des établissements d'enseignement et de recherche français ou étrangers, des laboratoires publics ou privés. 


\title{
Primal-dual splitting algorithm for solving inclusions with mixtures of composite, Lipschitzian, and parallel-sum type monotone operators*
}

\author{
Patrick L. Combettes ${ }^{1}$ and Jean-Christophe Pesquet ${ }^{2}$ \\ ${ }^{1}$ UPMC Université Paris 06 \\ Laboratoire Jacques-Louis Lions - UMR CNRS 7598 \\ 75005 Paris, France \\ plc@math.jussieu.fr \\ ${ }^{2}$ Université Paris-Est \\ Laboratoire d'Informatique Gaspard Monge - UMR CNRS 8049 \\ 77454 Marne la Vallée Cedex 2, France \\ jean-christophe.pesquet@univ-paris-est.fr
}

\begin{abstract}
We propose a primal-dual splitting algorithm for solving monotone inclusions involving a mixture of sums, linear compositions, and parallel sums of set-valued and Lipschitzian operators. An important feature of the algorithm is that the Lipschitzian operators present in the formulation can be processed individually via explicit steps, while the set-valued operators are processed individually via their resolvents. In addition, the algorithm is highly parallel in that most of its steps can be executed simultaneously. This work brings together and notably extends various types of structured monotone inclusion problems and their solution methods. The application to convex minimization problems is given special attention.
\end{abstract}

Keywords maximal monotone operator, monotone inclusion, nonsmooth convex optimization, parallel sum, set-valued duality, splitting algorithm

Mathematics Subject Classifications (2010) 47H05, 49M29, 49M27, 90C25, 49N15.

*Contact author: P. L. Combettes, plc@math.jussieu.fr, phone: +33 14427 6319, fax: +33 14427 7200. This work was supported by the Agence Nationale de la Recherche under grants ANR-08-BLAN-0294-02 and ANR-09EMER-004-03. 


\section{Introduction}

Duality theory occupies a central place in classical optimization $[19,24,33,40,41]$. Since the mid 1960s it has expanded in various directions, e.g., variational inequalities [2, 17, 21, 23, 26, 34], minimax and saddle point problems [27, 29, 32, 39], and, from a more global perspective, monotone inclusions $[5,9,10,16,31,37,38]$. In the present paper, we propose an algorithm for solving the following structured duality framework for monotone inclusions that encompasses the above cited works. In this formulation, we denote by $B \square D$ the parallel sum of two set-valued operators $B$ and $D$ (see (2.5)). This operation plays a central role in convex analysis and monotone operator theory. In particular, $B \square D$ can be seen as a regularization of $B$ by $D$, and $\square$ is naturally connected to addition through duality since $(B+D)^{-1}=B^{-1} \square D^{-1}$. It is also strongly related to the infimal convolution of functions through subdifferentials. We refer the reader to $[8,28,35,36,43]$ and the references therein for background on the parallel sum.

Problem 1.1 Let $\mathcal{H}$ be a real Hilbert space, let $z \in \mathcal{H}$, let $m$ be a strictly positive integer, let $A: \mathcal{H} \rightarrow 2^{\mathcal{H}}$ be maximally monotone, and let $C: \mathcal{H} \rightarrow \mathcal{H}$ be monotone and $\mu$-Lipschitzian for some $\mu \in] 0,+\infty\left[\right.$. For every $i \in\{1, \ldots, m\}$, let $\mathcal{G}_{i}$ be a real Hilbert space, let $r_{i} \in \mathcal{G}_{i}$, let $B_{i}: \mathcal{G}_{i} \rightarrow 2^{\mathcal{G}_{i}}$ be maximally monotone, let $D_{i}: \mathcal{G}_{i} \rightarrow 2^{\mathcal{G}_{i}}$ be monotone and such that $D_{i}^{-1}$ is $\nu_{i}$-Lipschitzian, for some $\left.\nu_{i} \in\right] 0,+\infty\left[\right.$, and suppose that $L_{i}: \mathcal{H} \rightarrow \mathcal{G}_{i}$ is a nonzero bounded linear operator. The problem is to solve the primal inclusion

$$
\text { find } \bar{x} \in \mathcal{H} \text { such that } z \in A \bar{x}+\sum_{i=1}^{m} L_{i}^{*}\left(\left(B_{i} \square D_{i}\right)\left(L_{i} \bar{x}-r_{i}\right)\right)+C \bar{x},
$$

together with the dual inclusion

$$
\text { find } \overline{v_{1}} \in \mathcal{G}_{1}, \ldots, \overline{v_{m}} \in \mathcal{G}_{m} \text { such that }(\exists x \in \mathcal{H})\left\{\begin{array}{l}
z-\sum_{i=1}^{m} L_{i}^{*} \overline{v_{i}} \in A x+C x \\
(\forall i \in\{1, \ldots, m\}) \overline{v_{i}} \in\left(B_{i} \square D_{i}\right)\left(L_{i} x-r_{i}\right) .
\end{array}\right.
$$

Problem 1.1 captures and extends various existing problem formulations. Here are some examples that illustrate its versatility and the breadth of its scope.

Example 1.2 In Problem 1.1 set

$$
m=1, \quad C: x \mapsto 0, \quad \text { and } \quad D_{1}: y \mapsto \begin{cases}\mathcal{G}_{1}, & \text { if } y=0 \\ \varnothing, & \text { if } y \neq 0 .\end{cases}
$$

Then we recover a duality framework investigated in $[10,16,37,38]$, namely (we drop the subscript ' 1 ' for brevity),

$$
\text { find }(\bar{x}, \bar{v}) \in \mathcal{H} \oplus \mathcal{G} \text { such that }\left\{\begin{array}{l}
z \in A \bar{x}+L^{*}(B(L \bar{x}-r)) \\
-r \in-L\left(A^{-1}\left(z-L^{*} \bar{v}\right)\right)+B^{-1} \bar{v}
\end{array}\right.
$$

Example 1.3 In Example 1.2, let $\mathcal{G}=\mathcal{H}, r=z=0$, and $L=\mathrm{Id}$. Then we obtain the duality setting of $[5,31]$, i.e.,

$$
\text { find }(\bar{x}, \bar{u}) \in \mathcal{H} \oplus \mathcal{H} \text { such that }\left\{\begin{array}{l}
0 \in A \bar{x}+B \bar{x} \\
0 \in-A^{-1}(-\bar{u})+B^{-1} \bar{u}
\end{array}\right.
$$


The special case of variational inequalities was first treated in [34].

Example 1.4 In Example 1.2, let $A$ and $B$ be the subdifferentials of lower semicontinuous convex functions $f: \mathcal{H} \rightarrow]-\infty,+\infty]$ and $g: \mathcal{G} \rightarrow]-\infty,+\infty]$, respectively. Then, under suitable constraint qualification, we obtain the classical Fenchel-Rockafellar duality framework [40], i.e.,

$$
\left\{\begin{array}{l}
\underset{x \in \mathcal{H}}{\operatorname{minimize}} f(x)+g(L x-r)-\langle x \mid z\rangle \\
\underset{v \in \mathcal{G}}{\operatorname{minimize}} f^{*}\left(z-L^{*} v\right)+g^{*}(v)+\langle v \mid r\rangle .
\end{array}\right.
$$

Example 1.5 In Problem 1.1, set $C: x \mapsto 0, z=0$, and $(\forall i \in\{1, \ldots, m\}) \mathcal{G}_{i}=\mathcal{H}, r_{i}=0, L_{i}=\mathrm{Id}$, and $D_{i}=\rho_{i}^{-1} \mathrm{Id}$, where $\left.\rho_{i} \in\right] 0,+\infty$ [. Then it follows from [8, Proposition 23.6(ii)] that, for every $i \in\{1, \ldots, m\}, B_{i} \square D_{i}=\left(\operatorname{Id}-J_{\rho_{i} B_{i}}\right) / \rho_{i}={ }^{\rho_{i}} B_{i}$ is the Yosida approximation of index $\rho_{i}$ of $B_{i}$. Thus, (1.1) reduces to

$$
\text { find } \bar{x} \in \mathcal{H} \text { such that } 0 \in A \bar{x}+\sum_{i=1}^{m}{ }^{\rho_{i}} B_{i} \bar{x} .
$$

This primal problem is investigated in [13, Section 6.3]. In the case when $m=1$, we obtain the primal-dual problem (we drop the subscript ' 1 ' for brevity)

$$
\text { find }(\bar{x}, \bar{u}) \in \mathcal{H} \oplus \mathcal{H} \text { such that }\left\{\begin{array}{l}
0 \in A \bar{x}+{ }^{\rho} B \bar{x} \\
0 \in-A^{-1}(-\bar{u})+B^{-1} \bar{u}+\rho \bar{u}
\end{array}\right.
$$

investigated in [9].

Example 1.6 In Problem 1.1, set $m=1, \mathcal{G}_{1}=\mathcal{G}, L_{1}=L, z=0$, and $r_{1}=0$, and let $A$ and $B_{1}$ be the subdifferentials of lower semicontinuous convex functions $f: \mathcal{H} \rightarrow]-\infty,+\infty]$ and $g: \mathcal{G} \rightarrow]-\infty,+\infty]$, respectively. In addition, let $C$ be the gradient of a differentiable convex function $h: \mathcal{H} \rightarrow \mathbb{R}$, and let $D$ be the subdifferential of a lower semicontinuous strongly convex function $\ell: \mathcal{G} \rightarrow]-\infty,+\infty]$. Then, under suitable constraint qualification, (1.1) assumes the form of the minimization problem

$$
\underset{x \in \mathcal{H}}{\operatorname{minimize}} f(x)+(g \square \ell)(L x)+h(x),
$$

which can be rewritten as

$$
\underset{x \in \mathcal{H}, y \in \mathcal{G}}{\operatorname{minimize}} f(x)+h(x)+g(y)+\ell(L x-y) .
$$

In the special case when $h=0, \mathcal{G}=\mathcal{H}, L=\mathrm{Id}$, and $\ell$ is a quadratic coupling function, such formulations have been investigated in $[1,4,6,12,15]$.

Example 1.7 In Problem 1.1, set $m=1, \mathcal{G}_{1}=\mathcal{H}, L_{1}=\mathrm{Id}, B_{1}=D_{1}^{-1}: x \mapsto\{0\}$, and $z=r_{1}=0$. Then (1.1) yields the inclusion $0 \in A \bar{x}+C \bar{x}$ studied in [45], where an algorithm using explicit steps for $C$ was proposed.

Example 1.8 In Problem 1.1, set $A: x \mapsto\{0\}$ and $C=\operatorname{Id}$. Furthermore, for every $i \in\{1, \ldots, m\}$, let $B_{i}$ be the subdifferential of a lower semicontinuous convex function $\left.\left.g_{i}: \mathcal{G}_{i} \rightarrow\right]-\infty,+\infty\right]$ and 
let $D_{i}^{-1}: y \mapsto\{0\}$. Then, under suitable constraint qualification, we obtain the primal-dual pair considered in [14], namely

$$
\underset{x \in \mathcal{H}}{\operatorname{minimize}} \sum_{i=1}^{m} g_{i}\left(L_{i} x-r_{i}\right)+\frac{1}{2}\|x-z\|^{2}
$$

and

$$
\underset{v_{1} \in \mathcal{G}_{1}, \ldots, v_{m} \in \mathcal{G}_{m}}{\operatorname{minimize}} \frac{1}{2}\left\|z-\sum_{i=1}^{m} L_{i}^{*} v_{i}\right\|^{2}+\sum_{i=1}^{m}\left(g_{i}^{*}\left(v_{i}\right)+\left\langle v_{i} \mid r_{i}\right\rangle\right)
$$

Example 1.9 The special case of Problem 1.1 in which

$$
A: x \mapsto\{0\}, C: x \mapsto 0, \quad \text { and } \quad(\forall i \in\{1, \ldots, m\}) \quad D_{i}: y \mapsto \begin{cases}\mathcal{G}_{i}, & \text { if } y=0 ; \\ \varnothing, & \text { if } y \neq 0\end{cases}
$$

yields the primal-dual pair

$$
\text { find } \bar{x} \in \mathcal{H} \text { such that } z \in \sum_{i=1}^{m} L_{i}^{*}\left(B_{i}\left(L_{i} \bar{x}-r_{i}\right)\right)
$$

and

$$
\text { find } \overline{v_{1}} \in \mathcal{G}_{1}, \ldots, \overline{v_{m}} \in \mathcal{G}_{m} \quad \text { such that }\left\{\begin{array}{l}
\sum_{i=1}^{m} L_{i}^{*} \overline{v_{i}}=z \\
(\exists x \in \mathcal{H})(\forall i \in\{1, \ldots, m\}) \overline{v_{i}} \in B_{i}\left(L_{i} x-r_{i}\right) .
\end{array}\right.
$$

This framework is considered in [10, Theorem 3.8].

Conceptually, the primal problem (1.1) could be recast in the form of (1.14), namely

$$
\text { find } \bar{x} \in \mathcal{H} \text { such that } z \in \sum_{i=0}^{m} L_{i}^{*}\left(E_{i}\left(L_{i} \bar{x}-r_{i}\right)\right) \text {, }
$$

where

$$
\mathcal{G}_{0}=\mathcal{H}, \quad E_{0}=A+C, L_{0}=\mathrm{Id}, r_{0}=0, \quad \text { and } \quad(\forall i \in\{1, \ldots, m\}) \quad E_{i}=B_{i} \square D_{i} .
$$

In turn, one could contemplate the possibility of using the primal-dual algorithm proposed in [10, Theorem 3.8] to solve Problem 1.1. However, this algorithm requires the computation of the resolvents of the operators $A+C$ and $\left(B_{i}^{-1}+D_{i}^{-1}\right)_{1 \leq i \leq m}$, which are usually intractable. Thus, for numerical purposes, Problem 1.1 cannot be reduced to Example 1.9. Let us stress that, even in the instance of the simple inclusion $0 \in A \bar{x}+C \bar{x}$, it is precisely the objective of the forward-backward splitting algorithm and its variants $[8,15,30,44,45]$ to circumvent the computation of the resolvent of $A+C$, as would impose a naive application of the proximal point algorithm [42].

The goal of this paper is to propose a fully split algorithm for solving Problem 1.1 that employs the operators $A,\left(L_{i}\right)_{1 \leq i \leq m},\left(B_{i}\right)_{1 \leq i \leq m},\left(D_{i}\right)_{1 \leq i \leq m}$, and $C$ separately. An important feature of the algorithm is to activate the single-valued operators $\left(L_{i}\right)_{1 \leq i \leq m},\left(D_{i}^{-1}\right)_{1 \leq i \leq m}$, and $C$ through explicit steps. In addition, it exhibits a highly parallel structure which allows for the simultaneous activation of the operators involved. This new splitting method goes significantly beyond the state-of-the-art, which is limited to specific subclasses of Problem 1.1.

In Section 2, we briefly set our notation. The new splitting method is proposed in Section 3, where we also prove its convergence. The special case of minimization problems is discussed in Section 4 . 


\section{Notation and background}

Our notation is standard. We refer the reader to $[8,46]$ for background on convex analysis and monotone operator theory. Hereafter, $\mathcal{K}$ is a real Hilbert space.

We denote the scalar product of a Hilbert space by $\langle\cdot \mid \cdot\rangle$ and the associated norm by $\|\cdot\|$. The symbols $\rightarrow$ and $\rightarrow$ denote respectively weak and strong convergence. Moreover, $\mathcal{G}_{1} \oplus \cdots \oplus \mathcal{G}_{m}$ is the Hilbert direct sum of the Hilbert spaces $\left(\mathcal{G}_{i}\right)_{1 \leq i \leq m}$ in Problem 1.1, i.e., their product space equipped with the norm $\left(y_{i}\right)_{1 \leq i \leq m} \mapsto \sqrt{\sum_{i=1}^{m}\left\|y_{i}\right\|^{2}}$. For every $i \in\{1, \ldots, m\}$, let $T_{i}$ be a mapping from $\mathcal{G}_{i}$ to some set $\mathcal{R}$. Then

$$
\bigoplus_{i=1}^{m} T_{i}: \bigoplus_{i=1}^{m} \mathcal{G}_{i} \rightarrow \mathcal{R}:\left(y_{i}\right)_{1 \leq i \leq m} \mapsto \sum_{i=1}^{m} T_{i} y_{i} .
$$

Let $M: \mathcal{K} \rightarrow 2^{\mathcal{K}}$ be a set-valued operator. We denote by $\operatorname{ran} M=\{u \in \mathcal{K} \mid(\exists x \in \mathcal{K}) u \in M x\}$ the range of $M$, by $\operatorname{dom} M=\{x \in \mathcal{K} \mid M x \neq \varnothing\}$ its domain, by zer $M=\{x \in \mathcal{K} \mid 0 \in M x\}$ its set of zeros, by gra $M=\{(x, u) \in \mathcal{K} \times \mathcal{K} \mid u \in M x\}$ its graph, and by $M^{-1}$ its inverse, i.e., the set-valued operator with graph $\{(u, x) \in \mathcal{K} \times \mathcal{K} \mid u \in M x\}$. The resolvent of $M$ is

$$
J_{M}=(\mathrm{Id}+M)^{-1},
$$

where Id denotes the identity operator on $\mathcal{K}$. Moreover, $M$ is monotone if

$$
(\forall(x, u) \in \operatorname{gra} M)(\forall(y, v) \in \operatorname{gra} M) \quad\langle x-y \mid u-v\rangle \geq 0,
$$

and maximally so if there exists no monotone operator $\widetilde{M}: \mathcal{K} \rightarrow 2^{\mathcal{K}}$ such that gra $M \subset$ gra $\widetilde{M} \neq$ gra $M$. We say that $M$ is uniformly monotone at $x \in \operatorname{dom} M$ if there exists an increasing function $\phi:[0,+\infty[\rightarrow[0,+\infty]$ that vanishes only at 0 such that

$$
(\forall u \in M x)(\forall(y, v) \in \operatorname{gra} M) \quad\langle x-y \mid u-v\rangle \geq \phi(\|x-y\|) .
$$

The parallel sum of two set-valued operators $M_{1}$ and $M_{2}$ from $\mathcal{K}$ to $2^{\mathcal{K}}$ is

$$
M_{1} \square M_{2}=\left(M_{1}^{-1}+M_{2}^{-1}\right)^{-1} .
$$

We denote by $\Gamma_{0}(\mathcal{K})$ the class of lower semicontinuous convex functions $\left.\left.\varphi: \mathcal{K} \rightarrow\right]-\infty,+\infty\right]$ such that $\operatorname{dom} \varphi=\{x \in \mathcal{K} \mid \varphi(x)<+\infty\} \neq \varnothing$. Now let $\varphi \in \Gamma_{0}(\mathcal{K})$. The conjugate of $\varphi$ is the function $\varphi^{*} \in \Gamma_{0}(\mathcal{K})$ defined by $\varphi^{*}: u \mapsto \sup _{x \in \mathcal{K}}(\langle x \mid u\rangle-\varphi(x))$, and the subdifferential of $\varphi$ is the maximally monotone operator

$$
\partial \varphi: \mathcal{K} \rightarrow 2^{\mathcal{K}}: x \mapsto\{u \in \mathcal{K} \mid(\forall y \in \mathcal{K})\langle y-x \mid u\rangle+\varphi(x) \leq \varphi(y)\}
$$

with inverse given by

$$
(\partial \varphi)^{-1}=\partial \varphi^{*} .
$$

Moreover, for every $x \in \mathcal{K}, \varphi+\|x-\cdot\|^{2} / 2$ possesses a unique minimizer, which is denoted by $\operatorname{prox}_{\varphi} x$. We have

$$
\operatorname{prox}_{\varphi}=J_{\partial \varphi} .
$$


We say that $\varphi$ is $\nu$-strongly convex for some $\nu \in] 0,+\infty\left[\right.$ if $\varphi-\nu\|\cdot\|^{2} / 2$ is convex, and that $\varphi$ is uniformly convex at $x \in \operatorname{dom} \varphi$ if there exists an increasing function $\phi:[0,+\infty[\rightarrow[0,+\infty]$ that vanishes only at 0 such that

$$
(\forall y \in \operatorname{dom} \varphi)(\forall \alpha \in] 0,1[) \quad \varphi(\alpha x+(1-\alpha) y)+\alpha(1-\alpha) \phi(\|x-y\|) \leq \alpha \varphi(x)+(1-\alpha) \varphi(y) .
$$

The infimal convolution of two functions $\varphi_{1}$ and $\varphi_{2}$ from $\mathcal{K}$ to $\left.]-\infty,+\infty\right]$ is

$$
\varphi_{1} \square \varphi_{2}: \mathcal{K} \rightarrow[-\infty,+\infty]: x \mapsto \inf _{y \in \mathcal{K}}\left(\varphi_{1}(y)+\varphi_{2}(x-y)\right) .
$$

Finally, let $S$ be a convex subset of $\mathcal{K}$. The strong relative interior of $S$, i.e., the set of points $x \in S$ such that the cone generated by $-x+S$ is a closed vector subspace of $\mathcal{K}$, is denoted by sri $S$, and the relative interior of $S$, i.e., the set of points $x \in S$ such that the cone generated by $-x+S$ is a vector subspace of $\mathcal{K}$, is denoted by ri $S$.

\section{Main result}

Our main result is the following theorem, which presents our new splitting algorithm and describes its asymptotic behavior.

Theorem 3.1 In Problem 1.1, suppose that

$$
z \in \operatorname{ran}\left(A+\sum_{i=1}^{m} L_{i}^{*}\left(B_{i} \square D_{i}\right)\left(L_{i} \cdot-r_{i}\right)+C\right) .
$$

Let $\left(a_{1, n}\right)_{n \in \mathbb{N}}, \quad\left(b_{1, n}\right)_{n \in \mathbb{N}}$, and $\left(c_{1, n}\right)_{n \in \mathbb{N}}$ be absolutely summable sequences in $\mathcal{H}$ and, for every $i \in\{1, \ldots, m\}$, let $\left(a_{2, i, n}\right)_{n \in \mathbb{N}},\left(b_{2, i, n}\right)_{n \in \mathbb{N}}$, and $\left(c_{2, i, n}\right)_{n \in \mathbb{N}}$ be absolutely summable sequences in $\mathcal{G}_{i}$. Furthermore, set

$$
\beta=\max \left\{\mu, \nu_{1}, \ldots, \nu_{m}\right\}+\sqrt{\sum_{i=1}^{m}\left\|L_{i}\right\|^{2}},
$$

let $x_{0} \in \mathcal{H}$, let $\left(v_{1,0}, \ldots, v_{m, 0}\right) \in \mathcal{G}_{1} \oplus \cdots \oplus \mathcal{G}_{m}$, let $\left.\varepsilon \in\right] 0,1 /(\beta+1)\left[\right.$, let $\left(\gamma_{n}\right)_{n \in \mathbb{N}}$ be a sequence in $[\varepsilon,(1-\varepsilon) / \beta]$, and set

$$
\begin{array}{l|l}
\forall n \in \mathbb{N}) \quad \mid \begin{array}{l}
y_{1, n}=x_{n}-\gamma_{n}\left(C x_{n}+\sum_{i=1}^{m} L_{i}^{*} v_{i, n}+a_{1, n}\right) \\
p_{1, n}=J_{\gamma_{n} A}\left(y_{1, n}+\gamma_{n} z\right)+b_{1, n}
\end{array} \\
\text { For } i=1, \ldots, m \\
\mid \begin{array}{l}
y_{2, i, n}=v_{i, n}+\gamma_{n}\left(L_{i} x_{n}-D_{i}^{-1} v_{i, n}+a_{2, i, n}\right) \\
p_{2, i, n}=J_{\gamma_{n} B_{i}^{-1}}\left(y_{2, i, n}-\gamma_{n} r_{i}\right)+b_{2, i, n} \\
q_{2, i, n}=p_{2, i, n}+\gamma_{n}\left(L_{i} p_{1, n}-D_{i}^{-1} p_{2, i, n}+c_{2, i, n}\right. \\
v_{i, n+1}=v_{i, n}-y_{2, i, n}+q_{2, i, n} .
\end{array} \\
q_{1, n}=p_{1, n}-\gamma_{n}\left(C p_{1, n}+\sum_{i=1}^{m} L_{i}^{*} p_{2, i, n}+c_{1, n}\right) \\
x_{n+1}=x_{n}-y_{1, n}+q_{1, n} .
\end{array}
$$

Then the following hold. 
(i) $\sum_{n \in \mathbb{N}}\left\|x_{n}-p_{1, n}\right\|^{2}<+\infty$ and $(\forall i \in\{1, \ldots, m\}) \sum_{n \in \mathbb{N}}\left\|v_{i, n}-p_{2, i, n}\right\|^{2}<+\infty$.

(ii) There exist a solution $\bar{x}$ to (1.1) and a solution $\left(\overline{v_{1}}, \ldots, \overline{v_{m}}\right)$ to $(1.2)$ such that the following hold.

(a) $z-\sum_{j=1}^{m} L_{j}^{*} \overline{v_{j}} \in A \bar{x}+C \bar{x}$ and $(\forall i \in\{1, \ldots, m\}) L_{i} \bar{x}-r_{i} \in B_{i}^{-1} \overline{v_{i}}+D_{i}^{-1} \overline{v_{i}}$.

(b) $(\forall i \in\{1, \ldots, m\})-r_{i} \in-L_{i}\left(\left(A^{-1} \square C^{-1}\right)\left(z-\sum_{j=1}^{m} L_{j}^{*} \overline{j_{j}}\right)\right)+B_{i}^{-1} \overline{v_{i}}+D_{i}^{-1} \overline{v_{i}}$.

(c) $x_{n} \rightarrow \bar{x}$ and $p_{1, n} \rightarrow \bar{x}$.

(d) $(\forall i \in\{1, \ldots, m\}) v_{i, n} \rightarrow \overline{v_{i}}$ and $p_{2, i, n} \rightarrow \overline{v_{i}}$.

(e) Suppose that $A$ or $C$ is uniformly monotone at $\bar{x}$. Then $x_{n} \rightarrow \bar{x}$ and $p_{1, n} \rightarrow \bar{x}$.

(f) Suppose that, for some $i \in\{1, \ldots, m\}, B_{i}^{-1}$ or $D_{i}^{-1}$ is uniformly monotone at $\overline{v_{i}}$. Then $v_{i, n} \rightarrow \overline{v_{i}}$ and $p_{2, i, n} \rightarrow \overline{v_{i}}$.

Proof. Let us first rewrite (3.3) as

$$
\begin{array}{l|l}
y_{1, n}=x_{n}-\gamma_{n}\left(C x_{n}+\sum_{i=1}^{m} L_{i}^{*} v_{i, n}+a_{1, n}\right) \\
\text { For } i=1, \ldots, m \\
\left\lfloor y_{2, i, n}=v_{i, n}+\gamma_{n}\left(L_{i} x_{n}-D_{i}^{-1} v_{i, n}+a_{2, i, n}\right)\right. \\
p_{1, n}=J_{\gamma_{n} A}\left(y_{1, n}+\gamma_{n} z\right)+b_{1, n} \\
\text { For } i=1, \ldots, m \\
\left\lfloor p_{2, i, n}=J_{\gamma_{n} B_{i}^{-1}}\left(y_{2, i, n}-\gamma_{n} r_{i}\right)+b_{2, i, n}\right. \\
q_{1, n}=p_{1, n}-\gamma_{n}\left(C p_{1, n}+\sum_{i=1}^{m} L_{i}^{*} p_{2, i, n}+c_{1, n}\right) \\
\text { For } i=1, \ldots, m \\
\left\lfloor q_{2, i, n}=p_{2, i, n}+\gamma_{n}\left(L_{i} p_{1, n}-D_{i}^{-1} p_{2, i, n}+c_{2, i, n}\right)\right. \\
x_{n+1}=x_{n}-y_{1, n}+q_{1, n} \\
\text { For } i=1, \ldots, m \\
\left\lfloor v_{i, n+1}=v_{i, n}-y_{2, i, n}+q_{2, i, n} .\right.
\end{array}
$$

Next, let us introduce the Hilbert space

$$
\mathcal{K}=\mathcal{H} \oplus \mathcal{G}_{1} \oplus \cdots \oplus \mathcal{G}_{m}
$$

and the operators

$$
\begin{aligned}
M: \mathcal{K} & \rightarrow 2^{\mathcal{K}} \\
\left(x, v_{1}, \ldots, v_{m}\right) & \mapsto(-z+A x) \times\left(r_{1}+B_{1}^{-1} v_{1}\right) \times \cdots \times\left(r_{m}+B_{m}^{-1} v_{m}\right)
\end{aligned}
$$

and

$$
\begin{aligned}
\boldsymbol{Q}: \mathcal{K} & \rightarrow \mathcal{K} \\
\left(x, v_{1}, \ldots, v_{m}\right) & \mapsto\left(C x+L_{1}^{*} v_{1}+\cdots+L_{m}^{*} v_{m},-L_{1} x+D_{1}^{-1} v_{1}, \ldots,-L_{m} x+D_{m}^{-1} v_{m}\right) .
\end{aligned}
$$

Since the operator $A$ and $\left(B_{i}\right)_{1 \leq i \leq m}$ are maximally monotone, so is $\boldsymbol{M}$ by [8, Propositions 20.22 and 20.23]. In addition, [8, Propositions 23.15(ii) and 23.16] yield

$$
\begin{aligned}
(\forall \gamma \in] 0,+\infty[) & \left(\forall\left(x, v_{1}, \ldots, v_{m}\right) \in \mathcal{K}\right) \\
& J_{\gamma M}\left(x, v_{1}, \ldots, v_{m}\right)=\left(J_{\gamma A}(x+\gamma z), J_{\gamma B_{1}^{-1}}\left(v_{1}-\gamma r_{1}\right), \ldots, J_{\gamma B_{m}^{-1}}\left(v_{m}-\gamma r_{m}\right)\right) .
\end{aligned}
$$


Let us now examine the properties of $\boldsymbol{Q}$. To this end, let $\left(x, v_{1}, \ldots, v_{m}\right)$ and $\left(y, w_{1}, \ldots, w_{m}\right)$ be two points in $\mathcal{K}$. Using the monotonicity of the operators $C$ and $\left(D_{i}^{-1}\right)_{1 \leq i \leq m}$, we derive from (3.7) that

$$
\begin{aligned}
\left\langle\left(x, v_{1}, \ldots, v_{m}\right)-\left(y, w_{1}, \ldots, w_{m}\right) \mid \boldsymbol{Q}\left(x, v_{1}, \ldots, v_{m}\right)-\boldsymbol{Q}\left(y, w_{1}, \ldots, w_{m}\right)\right\rangle & \\
= & \left\langle\left(x-y, v_{1}-w_{1}, \ldots, v_{m}-w_{m}\right)\right|\left(C x-C y+L_{1}^{*}\left(v_{1}-w_{1}\right)+\cdots+L_{m}^{*}\left(v_{m}-w_{m}\right),\right. \\
& \left.\left.\quad-L_{1}(x-y)+D_{1}^{-1} v_{1}-D_{1}^{-1} w_{1}, \ldots,-L_{m}(x-y)+D_{m}^{-1} v_{m}-D_{m}^{-1} w_{m}\right)\right\rangle \\
= & \langle x-y \mid C x-C y\rangle+\sum_{i=1}^{m}\left\langle v_{i}-w_{i} \mid D_{i}^{-1} v_{i}-D_{i}^{-1} w_{i}\right\rangle \\
& \quad+\sum_{i=1}^{m}\left(\left\langle x-y \mid L_{i}^{*}\left(v_{i}-w_{i}\right)\right\rangle-\left\langle v_{i}-w_{i} \mid L_{i}(x-y)\right\rangle\right) \\
= & \langle x-y \mid C x-C y\rangle+\sum_{i=1}^{m}\left\langle v_{i}-w_{i} \mid D_{i}^{-1} v_{i}-D_{i}^{-1} w_{i}\right\rangle \\
\geq & 0 .
\end{aligned}
$$

Hence, $\boldsymbol{Q}$ is monotone. Using the triangle inequality, the Lipschitzianity assumptions, the CauchySchwarz inequality, and (3.2), we obtain

$$
\begin{aligned}
& \left\|\boldsymbol{Q}\left(x, v_{1}, \ldots, v_{m}\right)-\boldsymbol{Q}\left(y, w_{1}, \ldots, w_{m}\right)\right\| \\
& =\|\left(C x-C y, D_{1}^{-1} v_{1}-D_{1}^{-1} w_{1}, \ldots, D_{m}^{-1} v_{m}-D_{m}^{-1} w_{m}\right) \\
& +\left(\sum_{i=1}^{m} L_{i}^{*}\left(v_{i}-w_{i}\right),-L_{1}(x-y), \ldots,-L_{m}(x-y)\right) \| \\
& \leq\left\|\left(C x-C y, D_{1}^{-1} v_{1}-D_{1}^{-1} w_{1}, \ldots, D_{m}^{-1} v_{m}-D_{m}^{-1} w_{m}\right)\right\| \\
& +\left\|\left(\sum_{i=1}^{m} L_{i}^{*}\left(v_{i}-w_{i}\right),-L_{1}(x-y), \ldots,-L_{m}(x-y)\right)\right\| \\
& =\sqrt{\|C x-C y\|^{2}+\sum_{i=1}^{m}\left\|D_{i}^{-1} v_{i}-D_{i}^{-1} w_{i}\right\|^{2}}+\sqrt{\left\|\sum_{i=1}^{m} L_{i}^{*}\left(v_{i}-w_{i}\right)\right\|^{2}+\sum_{i=1}^{m}\left\|L_{i}(x-y)\right\|^{2}} \\
& \leq \sqrt{\mu^{2}\|x-y\|^{2}+\sum_{i=1}^{m} \nu_{i}^{2}\left\|v_{i}-w_{i}\right\|^{2}}+\sqrt{\left(\sum_{i=1}^{m}\left\|L_{i}\right\|\left\|v_{i}-w_{i}\right\|\right)^{2}+\sum_{i=1}^{m}\left\|L_{i}\right\|^{2}\|x-y\|^{2}} \\
& \leq \max \left\{\mu, \nu_{1}, \ldots, \nu_{m}\right\} \sqrt{\|x-y\|^{2}+\sum_{i=1}^{m}\left\|v_{i}-w_{i}\right\|^{2}} \\
& +\sqrt{\left(\sum_{i=1}^{m}\left\|L_{i}\right\|^{2}\right)\left(\sum_{i=1}^{m}\left\|v_{i}-w_{i}\right\|^{2}\right)+\left(\sum_{i=1}^{m}\left\|L_{i}\right\|^{2}\right)\|x-y\|^{2}} \\
& =\beta\left\|\left(x, v_{1}, \ldots, v_{m}\right)-\left(y, w_{1}, \ldots, w_{m}\right)\right\| .
\end{aligned}
$$

To sum up, we have shown that

$\boldsymbol{M}$ is maximally monotone and $\boldsymbol{Q}$ is monotone and $\beta$-Lipschitzian. 
Next, let us observe that

$$
\begin{aligned}
&(3.1) \Leftrightarrow(\exists x \in \mathcal{H}) \quad z \in A x+\sum_{i=1}^{m} L_{i}^{*}\left(\left(B_{i} \square D_{i}\right)\left(L_{i} x-r_{i}\right)\right)+C x \\
& \Leftrightarrow\left(\exists\left(x, v_{1}, \ldots, v_{m}\right) \in \mathcal{K}\right)\left\{\begin{array}{c}
z \in A x+\sum_{i=1}^{m} L_{i}^{*} v_{i}+C x \\
v_{1} \in\left(B_{1} \square D_{1}\right)\left(L_{1} x-r_{1}\right) \\
\vdots \\
v_{m} \in\left(B_{m} \square D_{m}\right)\left(L_{m} x-r_{m}\right)
\end{array}\right. \\
& \Leftrightarrow\left(\exists\left(x, v_{1}, \ldots, v_{m}\right) \in \mathcal{K}\right)\left\{\begin{array}{c}
0 \in-z+A x+\sum_{i=1}^{m} L_{i}^{*} v_{i}+C x \\
0 \in r_{1}+B_{1}^{-1} v_{1}+D_{1}^{-1} v_{1}-L_{1} x \\
\vdots \\
0 \in r_{m}+B_{m}^{-1} v_{m}+D_{m}^{-1} v_{m}-L_{m} x
\end{array}\right. \\
& \Leftrightarrow\left(\exists\left(x, v_{1}, \ldots, v_{m}\right) \in \mathcal{K}\right) \quad(0, \ldots, 0) \in(-z+A x) \times\left(r_{1}+B_{1}^{-1} v_{1}\right) \times \cdots \times\left(r_{m}+B_{m}^{-1} v_{m}\right)
\end{aligned}
$$

In other words,

$$
\operatorname{zer}(\boldsymbol{M}+\boldsymbol{Q}) \neq \varnothing
$$

Now, let us set

$$
(\forall n \in \mathbb{N}) \quad\left\{\begin{array} { l } 
{ \boldsymbol { x } _ { n } = ( x _ { n } , v _ { 1 , n } , \ldots , v _ { 1 , n } ) } \\
{ \boldsymbol { y } _ { n } = ( y _ { 1 , n } , y _ { 2 , 1 , n } , \ldots , y _ { 2 , m , n } ) } \\
{ \boldsymbol { p } _ { n } = ( p _ { 1 , n } , p _ { 2 , 1 , n } , \ldots , p _ { 2 , m , n } ) } \\
{ \boldsymbol { q } _ { n } = ( q _ { 1 , n } , q _ { 2 , 1 , n } , \ldots , q _ { 2 , m , n } ) }
\end{array} \quad \text { and } \quad \left\{\begin{array}{l}
\boldsymbol{a}_{n}=\left(a_{1, n}, a_{2,1, n}, \ldots, a_{2, m, n}\right) \\
\boldsymbol{b}_{n}=\left(b_{1, n}, b_{2,1, n}, \ldots, b_{2, m, n}\right) \\
\boldsymbol{c}_{n}=\left(c_{1, n}, c_{2,1, n}, \ldots, c_{2, m, n}\right) .
\end{array}\right.\right.
$$

We first observe that our assumptions imply that

$$
\sum_{n \in \mathbb{N}}\left\|\boldsymbol{a}_{n}\right\|<+\infty, \quad \sum_{n \in \mathbb{N}}\left\|\boldsymbol{b}_{n}\right\|<+\infty, \quad \text { and } \quad \sum_{n \in \mathbb{N}}\left\|\boldsymbol{c}_{n}\right\|<+\infty .
$$

Furthermore, it follows from (3.7), (3.8), and (3.14), that (3.4) assumes in $\mathcal{K}$ the form of the errortolerant forward-backward-forward algorithm

$$
(\forall n \in \mathbb{N}) \quad \mid \begin{aligned}
& \boldsymbol{y}_{n}=\boldsymbol{x}_{n}-\gamma_{n}\left(\boldsymbol{Q} \boldsymbol{x}_{n}+\boldsymbol{a}_{n}\right) \\
& \boldsymbol{p}_{n}=J_{\gamma_{n}} \boldsymbol{M} \boldsymbol{y}_{n}+\boldsymbol{b}_{n} \\
& \boldsymbol{q}_{n}=\boldsymbol{p}_{n}-\gamma_{n}\left(\boldsymbol{Q} \boldsymbol{p}_{n}+\boldsymbol{c}_{n}\right) \\
& \boldsymbol{x}_{n+1}=\boldsymbol{x}_{n}-\boldsymbol{y}_{n}+\boldsymbol{q}_{n}
\end{aligned}
$$

(i): It follows from (3.11), (3.13), (3.15), (3.16), and [10, Theorem 2.5(i)] that $\sum_{n \in \mathbb{N}}\left\|\boldsymbol{x}_{n}-\boldsymbol{p}_{n}\right\|^{2}<$ $+\infty$

(ii): It follows from [10, Theorem 2.5(ii)] that there exists $\overline{\boldsymbol{x}} \in \operatorname{zer}(\boldsymbol{M}+\boldsymbol{Q})$ such that

$$
\boldsymbol{x}_{n} \rightarrow \overline{\boldsymbol{x}} \text { and } \boldsymbol{p}_{n} \rightarrow \overline{\boldsymbol{x}} .
$$


Let us set

$$
\overline{\boldsymbol{x}}=\left(\bar{x}, \overline{v_{1}}, \ldots, \overline{v_{m}}\right)
$$

In view of (3.6) and (3.7),

$$
\begin{aligned}
& \overline{\boldsymbol{x}} \in \operatorname{zer}(\boldsymbol{M}+\boldsymbol{Q}) \Leftrightarrow\left\{\begin{aligned}
0 & \in-z+A \bar{x}+\sum_{i=1}^{m} L_{i}^{*} \overline{v_{i}}+C \bar{x} \\
0 & \in r_{1}+B_{1}^{-1} \overline{v_{1}}+D_{1}^{-1} \overline{v_{1}}-L_{1} \bar{x} \\
\vdots & \\
0 & \in r_{m}+B_{m}^{-1} \overline{v_{m}}+D_{m}^{-1} \overline{v_{m}}-L_{m} \bar{x}
\end{aligned}\right. \\
& \Leftrightarrow\left\{\begin{array}{c}
z-\sum_{j=1}^{m} L_{j}^{*} \overline{v_{j}} \in A \bar{x}+C \bar{x} \\
L_{1} \bar{x}-r_{1} \in\left(B_{1}^{-1}+D_{1}^{-1}\right) \overline{v_{1}} \\
\vdots \\
L_{m} \bar{x}-r_{m} \in\left(B_{m}^{-1}+D_{m}^{-1}\right) \overline{v_{m}}
\end{array}\right. \\
& \Leftrightarrow\left\{\begin{array}{l}
z-\sum_{j=1}^{m} L_{j}^{*} \overline{v_{j}} \in A \bar{x}+C \bar{x} \\
\overline{v_{1}} \in\left(B_{1} \square D_{1}\right)\left(L_{1} \bar{x}-r_{1}\right) \\
\vdots \\
\overline{v_{m}} \in\left(B_{m} \square D_{m}\right)\left(L_{m} \bar{x}-r_{m}\right)
\end{array}\right. \\
& \Rightarrow\left\{\begin{array}{l}
z-\sum_{j=1}^{m} L_{j}^{*} \overline{v_{j}} \in A \bar{x}+C \bar{x} \\
L_{1}^{*} \overline{v_{1}} \in L_{1}^{*}\left(\left(B_{1} \square D_{1}\right)\left(L_{1} \bar{x}-r_{1}\right)\right) \\
\vdots \\
L_{m}^{*} \overline{v_{m}} \in L_{m}^{*}\left(\left(B_{m} \square D_{m}\right)\left(L_{m} \bar{x}-r_{m}\right)\right)
\end{array}\right. \\
& \Rightarrow z \in A \bar{x}+\sum_{i=1}^{m} L_{i}^{*}\left(\left(B_{i} \square D_{i}\right)\left(L_{i} \bar{x}-r_{i}\right)\right)+C \bar{x} \\
& \Leftrightarrow \bar{x} \text { solves }(1.1) \text {. }
\end{aligned}
$$

On the other hand, (3.20) means that

$$
\left(\overline{v_{1}}, \ldots, \overline{v_{m}}\right) \text { solves }(1.2)
$$

(ii)(a): This follows from (3.19).

(ii)(b): We derive from (3.19) that

$$
\bar{x} \in(A+C)^{-1}\left(z-\sum_{j=1}^{m} L_{j}^{*} \overline{v_{j}}\right) \quad \text { and } \quad(\forall i \in\{1, \ldots, m\}) \quad L_{i} \bar{x}-r_{i} \in\left(B_{i}^{-1}+D_{i}^{-1}\right) \overline{v_{i}} .
$$

Hence,

$$
(\forall i \in\{1, \ldots, m\}) \quad\left\{\begin{array}{l}
-L_{i} \bar{x} \in-L_{i}\left(\left(A^{-1} \square C^{-1}\right)\left(z-\sum_{j=1}^{m} L_{j}^{*} \overline{v_{j}}\right)\right) \\
L_{i} \bar{x}-r_{i} \in\left(B_{i}^{-1}+D_{i}^{-1}\right) \overline{v_{i}}
\end{array}\right.
$$

Thus,

$$
(\forall i \in\{1, \ldots, m\}) \quad-r_{i} \in-L_{i}\left(\left(A^{-1} \square C^{-1}\right)\left(z-\sum_{j=1}^{m} L_{j}^{*} \overline{v_{j}}\right)\right)+B_{i}^{-1} \overline{v_{i}}+D_{i}^{-1} \overline{v_{i}} .
$$


(ii)(c): This follows from (3.17), (3.18), and (3.21).

(ii)(d): This follows from (3.17), (3.18), and (3.22).

(ii)(e): Let us set

$$
(\forall n \in \mathbb{N}) \quad\left\{\begin{array}{l}
\widetilde{y}_{1, n}=x_{n}-\gamma_{n}\left(C x_{n}+\sum_{j=1}^{m} L_{j}^{*} v_{j, n}\right) \\
\widetilde{p}_{1, n}=J_{\gamma_{n} A}\left(\widetilde{y}_{1, n}+\gamma_{n} z\right)
\end{array}\right.
$$

and

$$
(\forall i \in\{1, \ldots, m\})(\forall n \in \mathbb{N}) \quad\left\{\begin{array}{l}
\widetilde{y}_{2, i, n}=v_{i, n}+\gamma_{n}\left(L_{i} x_{n}-D_{i}^{-1} v_{i, n}\right) \\
\widetilde{p}_{2, i, n}=J_{\gamma_{n} B_{i}^{-1}}\left(\widetilde{y}_{2, i, n}-\gamma_{n} r_{i}\right) .
\end{array}\right.
$$

Then, in view of (3.3),

$$
(\forall n \in \mathbb{N}) \quad\left\|y_{1, n}-\widetilde{y}_{1, n}\right\| \leq \gamma_{n}\left\|a_{1, n}\right\| \leq \beta^{-1}\left\|a_{1, n}\right\|
$$

and, using the nonexpansiveness of the resolvents [8, Proposition 23.7], we obtain

$$
\begin{aligned}
(\forall n \in \mathbb{N}) \quad\left\|p_{1, n}-\widetilde{p}_{1, n}\right\| & \leq\left\|J_{\gamma_{n} A}\left(y_{1, n}+\gamma_{n} z\right)+b_{1, n}-J_{\gamma_{n} A}\left(\widetilde{y}_{1, n}+\gamma_{n} z\right)\right\| \\
& \leq\left\|y_{1, n}-\widetilde{y}_{1, n}\right\|+\left\|b_{1, n}\right\| \\
& \leq \beta^{-1}\left\|a_{1, n}\right\|+\left\|b_{1, n}\right\| .
\end{aligned}
$$

Since the sequences $\left(a_{1, n}\right)_{n \in \mathbb{N}}$ and $\left(b_{1, n}\right)_{n \in \mathbb{N}}$ are absolutely summable, it follows that

$$
y_{1, n}-\widetilde{y}_{1, n} \rightarrow 0 \quad \text { and } \quad p_{1, n}-\widetilde{p}_{1, n} \rightarrow 0 .
$$

Using the same arguments, we derive from (3.3) and (3.27) that

$$
(\forall i \in\{1, \ldots, m\}) \quad y_{2, i, n}-\widetilde{y}_{2, i, n} \rightarrow 0 \quad \text { and } \quad p_{2, i, n}-\widetilde{p}_{2, i, n} \rightarrow 0 .
$$

On the other hand, we deduce from (ii)(a) that there exists $u \in \mathcal{H}$ such that

$$
u \in A \bar{x} \quad \text { and } \quad z=u+\sum_{j=1}^{m} L_{j}^{*} \overline{v_{j}}+C \bar{x}
$$

and that

$$
(\forall i \in\{1, \ldots, m\}) \quad L_{i} \bar{x}-r_{i}-D_{i}^{-1} \overline{v_{i}} \in B_{i}^{-1} \overline{v_{i}} .
$$

In addition, (3.26) yields

$$
(\forall n \in \mathbb{N}) \quad \gamma_{n}^{-1}\left(x_{n}-\widetilde{p}_{1, n}\right)-C x_{n}-\sum_{j=1}^{m} L_{j}^{*} v_{j, n}+z \in A \widetilde{p}_{1, n}
$$

while (3.27) yields

$$
(\forall i \in\{1, \ldots, m\})(\forall n \in \mathbb{N}) \quad \gamma_{n}^{-1}\left(v_{i, n}-\widetilde{p}_{2, i, n}\right)+L_{i} x_{n}-D_{i}^{-1} v_{i, n}-r_{i} \in B_{i}^{-1} \widetilde{p}_{2, i, n} .
$$

Now let us set

$$
(\forall n \in \mathbb{N}) \quad\left\{\begin{array}{l}
\alpha_{1, n}=\left\|x_{n}-\widetilde{p}_{1, n}\right\|\left(\varepsilon^{-1}\left\|\widetilde{p}_{1, n}-\bar{x}\right\|+\mu\left\|x_{n}-\bar{x}\right\|+\sum_{i=1}^{m}\left\|L_{i}\right\|\left\|v_{i, n}-\overline{v_{i}}\right\|\right) \\
\alpha_{2, n}=\sum_{i=1}^{m}\left(\varepsilon^{-1}+\nu_{i}\right)\left\|v_{i, n}-\widetilde{p}_{2, i, n}\right\|\left\|\widetilde{p}_{2, i, n}-\overline{v_{i}}\right\| .
\end{array}\right.
$$


It follows from (i), (ii)(c), (ii)(d), (3.30), and (3.31) that

$$
\alpha_{1, n} \rightarrow 0 \quad \text { and } \quad \alpha_{2, n} \rightarrow 0 .
$$

Using the Cauchy-Schwarz inequality, and the Lipschitzianity and monotonicity of $C$, we obtain

$$
\begin{aligned}
& (\forall n \in \mathbb{N}) \quad \alpha_{1, n}+\left\langle x_{n}-\bar{x} \mid \sum_{i=1}^{m} L_{i}^{*}\left(\overline{v_{i}}-v_{i, n}\right)\right\rangle \\
& \geq\left\|x_{n}-\widetilde{p}_{1, n}\right\|\left(\varepsilon^{-1}\left\|\widetilde{p}_{1, n}-\bar{x}\right\|+\left\|C x_{n}-C \bar{x}\right\|\right)+\left\langle\widetilde{p}_{1, n}-x_{n} \mid \sum_{i=1}^{m} L_{i}^{*}\left(\bar{v}_{i}-v_{i, n}\right)\right\rangle \\
& +\left\langle x_{n}-\bar{x} \mid \sum_{i=1}^{m} L_{i}^{*}\left(\overline{v_{i}}-v_{i, n}\right)\right\rangle \\
& =\left\|x_{n}-\widetilde{p}_{1, n}\right\|\left(\varepsilon^{-1}\left\|\widetilde{p}_{1, n}-\bar{x}\right\|+\left\|C x_{n}-C \bar{x}\right\|\right)+\left\langle\widetilde{p}_{1, n}-\bar{x} \mid \sum_{i=1}^{m} L_{i}^{*}\left(\overline{v_{i}}-v_{i, n}\right)\right\rangle \\
& \geq\left\langle\widetilde{p}_{1, n}-\bar{x} \mid \gamma_{n}^{-1}\left(x_{n}-\widetilde{p}_{1, n}\right)+\sum_{i=1}^{m} L_{i}^{*}\left(\overline{v_{i}}-v_{i, n}\right)\right\rangle+\left\langle\widetilde{p}_{1, n}-x_{n} \mid C \bar{x}-C x_{n}\right\rangle \\
& =\left\langle\widetilde{p}_{1, n}-\bar{x} \mid \gamma_{n}^{-1}\left(x_{n}-\widetilde{p}_{1, n}\right)-\sum_{i=1}^{m} L_{i}^{*} v_{i, n}-C x_{n}+\sum_{i=1}^{m} L_{i}^{*} \overline{v_{i}}+C \bar{x}\right\rangle+\left\langle\bar{x}-x_{n} \mid C \bar{x}-C x_{n}\right\rangle \\
& =\left\langle\widetilde{p}_{1, n}-\bar{x} \mid \gamma_{n}^{-1}\left(x_{n}-\widetilde{p}_{1, n}\right)-\sum_{i=1}^{m} L_{i}^{*} v_{i, n}-C x_{n}+z-u\right\rangle+\left\langle\bar{x}-x_{n} \mid C \bar{x}-C x_{n}\right\rangle \\
& \geq\left\langle\widetilde{p}_{1, n}-\bar{x} \mid\left(\gamma_{n}^{-1}\left(x_{n}-\widetilde{p}_{1, n}\right)-\sum_{i=1}^{m} L_{i}^{*} v_{i, n}-C x_{n}+z\right)-u\right\rangle .
\end{aligned}
$$

Now suppose that $A$ is uniformly monotone at $\bar{x}$. Then, in view of (3.32), (3.34), and (3.38), there exists an increasing function $\phi_{A}:[0,+\infty[\rightarrow[0,+\infty]$ that vanishes only at 0 such that

$$
(\forall n \in \mathbb{N}) \quad \alpha_{1, n}+\left\langle x_{n}-\bar{x} \mid \sum_{i=1}^{m} L_{i}^{*}\left(\overline{v_{i}}-v_{i, n}\right)\right\rangle \geq \phi_{A}\left(\left\|\widetilde{p}_{1, n}-\bar{x}\right\|\right) .
$$

On the other hand, it follows from (3.36), the Lipschitzianity of the operators $\left(D_{i}^{-1}\right)_{1 \leq i \leq m},(3.33)$, (3.35), and the monotonicity of the operators $\left(B_{i}^{-1}\right)_{1 \leq i \leq m}$ and $\left(D_{i}^{-1}\right)_{1 \leq i \leq m}$ that

$$
\begin{aligned}
(\forall n \in \mathbb{N}) & \alpha_{2, n}+\left\langle x_{n}-\bar{x} \mid \sum_{i=1}^{m} L_{i}^{*}\left(\widetilde{p}_{2, i, n}-\overline{v_{i}}\right)\right\rangle \\
\geq & \sum_{i=1}^{m}\left\langle\gamma_{n}^{-1}\left(v_{i, n}-\widetilde{p}_{2, i, n}\right)-D_{i}^{-1} v_{i, n}+D_{i}^{-1} \widetilde{p}_{2, i, n}+L_{i}\left(x_{n}-\bar{x}\right) \mid \widetilde{p}_{2, i, n}-\overline{v_{i}}\right\rangle \\
= & \sum_{i=1}^{m}\left(\left\langle\gamma_{n}^{-1}\left(v_{i, n}-\widetilde{p}_{2, i, n}\right)+L_{i} x_{n}-D_{i}^{-1} v_{i, n}-r_{i}-\left(L_{i} \bar{x}-r_{i}-D_{i}^{-1} \bar{v}_{i}\right) \mid \widetilde{p}_{2, i, n}-\overline{v_{i}}\right\rangle\right. \\
& \left.\quad+\left\langle D_{i}^{-1} \widetilde{p}_{2, i, n}-D_{i}^{-1} \bar{v}_{i} \mid \widetilde{p}_{2, i, n}-\overline{v_{i}}\right\rangle\right) \\
\geq & 0 .
\end{aligned}
$$


Adding (3.39) and (3.40) yields

$$
(\forall n \in \mathbb{N}) \quad \alpha_{1, n}+\alpha_{2, n}+\left\langle x_{n}-\bar{x} \mid \sum_{i=1}^{m} L_{i}^{*}\left(\widetilde{p}_{2, i, n}-v_{i, n}\right)\right\rangle \geq \phi_{A}\left(\left\|\widetilde{p}_{1, n}-\bar{x}\right\|\right) .
$$

It then follows from (3.37), (ii)(c), (i), (3.31), and [8, Lemma 2.41(iii)] that $\phi_{A}\left(\left\|\widetilde{p}_{1, n}-\bar{x}\right\|\right) \rightarrow 0$ and, in turn, that $\widetilde{p}_{1, n} \rightarrow \bar{x}$. Hence, in view of (i) and (3.30), we get $x_{n} \rightarrow \bar{x}$ and $p_{1, n} \rightarrow \bar{x}$. Likewise, if $C$ is uniformly monotone at $\bar{x}$, there exists an increasing function $\phi_{C}:[0,+\infty[\rightarrow[0,+\infty]$ that vanishes only at 0 such that

$$
(\forall n \in \mathbb{N}) \quad \alpha_{1, n}+\alpha_{2, n}+\left\langle x_{n}-\bar{x} \mid \sum_{i=1}^{m} L_{i}^{*}\left(\widetilde{p}_{2, i, n}-v_{i, n}\right)\right\rangle \geq \phi_{C}\left(\left\|x_{n}-\bar{x}\right\|\right),
$$

and we reach the same conclusion.

(ii)(f): Suppose that $B_{i}^{-1}$ is uniformly monotone at $\overline{v_{i}}$ for some $i \in\{1, \ldots, m\}$. Then, proceeding as in (3.40), there exists an increasing function $\phi_{B_{i}}:[0,+\infty[\rightarrow[0,+\infty]$ that vanishes only at 0 such that

$$
\begin{aligned}
& (\forall n \in \mathbb{N}) \quad \alpha_{2, n}+\left\langle x_{n}-\bar{x} \mid \sum_{j=1}^{m} L_{j}^{*}\left(\widetilde{p}_{2, j, n}-\overline{v_{j}}\right)\right\rangle \\
& \geq \sum_{j=1}^{m}\left(\left\langle\gamma_{n}^{-1}\left(v_{j, n}-\widetilde{p}_{2, j, n}\right)+L_{j} x_{n}-D_{j}^{-1} v_{j, n}-r_{j}-\left(L_{j} \bar{x}-r_{j}-D_{j}^{-1} \overline{v_{j}}\right) \mid \widetilde{p}_{2, j, n}-\overline{v_{j}}\right\rangle\right. \\
& \left.\quad+\left\langle D_{j}^{-1} \widetilde{p}_{2, j, n}-D_{j}^{-1} \overline{v_{j}} \mid \widetilde{p}_{2, j, n}-\overline{v_{j}}\right\rangle\right) \\
& \quad \\
& \geq \sum_{j=1}^{m}\left\langle\gamma_{n}^{-1}\left(v_{j, n}-\widetilde{p}_{2, j, n}\right)+L_{j} x_{n}-D_{j}^{-1} v_{j, n}-r_{j}-\left(L_{j} \bar{x}-r_{j}-D_{j}^{-1} \overline{v_{j}}\right) \mid \widetilde{p}_{2, j, n}-\overline{v_{j}}\right\rangle \\
& \geq\left\langle\gamma_{n}^{-1}\left(v_{i, n}-\widetilde{p}_{2, i, n}\right)+L_{i} x_{n}-D_{i}^{-1} v_{i, n}-r_{i}-\left(L_{i} \bar{x}-r_{i}-D_{i}^{-1} \bar{v}_{i}\right) \mid \widetilde{p}_{2, i, n}-\overline{v_{i}}\right\rangle \\
& \geq \phi_{B_{i}}\left(\left\|\widetilde{p}_{2, i, n}-\bar{v}_{i}\right\|\right) .
\end{aligned}
$$

On the other hand, according to (3.38),

$$
(\forall n \in \mathbb{N}) \quad \alpha_{1, n}+\left\langle x_{n}-\bar{x} \mid \sum_{j=1}^{m} L_{j}^{*}\left(\overline{v_{j}}-v_{j, n}\right)\right\rangle \geq 0 .
$$

Hence,

$$
(\forall n \in \mathbb{N}) \quad \alpha_{1, n}+\alpha_{2, n}+\left\langle x_{n}-\bar{x} \mid \sum_{j=1}^{m} L_{j}^{*}\left(\widetilde{p}_{2, j, n}-v_{j, n}\right)\right\rangle \geq \phi_{B_{i}}\left(\left\|\widetilde{p}_{2, i, n}-\overline{v_{i}}\right\|\right) .
$$

By proceeding as previously, we infer that $\widetilde{p}_{2, i, n} \rightarrow \overline{v_{i}}$ and hence, via (3.31) and (i), that $p_{2, i, n} \rightarrow \overline{v_{i}}$ and $v_{i, n} \rightarrow \overline{v_{i}}$. If $D_{i}^{-1}$ is uniformly monotone at $\overline{v_{i}}$, the same arguments lead to these conclusions.

In the following remarks, we comment on the structure of the proposed algorithm and its relation to existing work. 
Remark 3.2 Here are some observations regarding the structure of algorithm (3.3).

(i) The algorithm achieves full splitting in that each of the operators appearing in Problem 1.1 is used separately.

(ii) The algorithm uses explicit steps for the single-valued operators and implicit steps for the set-valued operators. Since explicit steps are typically much easier to implement than implicit steps, the algorithm therefore exploits efficiently the properties of the operators.

(iii) The sequences $\left(a_{1, n}\right)_{n \in \mathbb{N}},\left(b_{1, n}\right)_{n \in \mathbb{N}}$, and $\left(c_{1, n}\right)_{n \in \mathbb{N}}$, and, for every $i \in\{1, \ldots, m\},\left(a_{2, i, n}\right)_{n \in \mathbb{N}}$, $\left(b_{2, i, n}\right)_{n \in \mathbb{N}}$, and $\left(c_{2, i, n}\right)_{n \in \mathbb{N}}$ relax the requirement for exact evaluations of the operators over the course of the iterations.

(iv) Most of the elementary steps in (3.3) can be executed in parallel.

(v) The update of the variable $p_{2, i, n}$ can also be carried out using the resolvent of $B_{i}$ since [8, Proposition 23.18] $J_{\gamma_{n} B_{i}^{-1}}\left(y_{2, i, n}-\gamma_{n} r_{i}\right)=y_{2, i, n}-\gamma_{n} r_{i}-\gamma_{n} J_{\gamma_{n}^{-1} B_{i}}\left(\gamma_{n}^{-1}\left(y_{2, i, n}-\gamma_{n} r_{i}\right)\right)$.

Remark 3.3 Some noteworthy connections between Theorem 3.1 and existing work are the following.

(i) Unlike most splitting methods, the proposed algorithm is designed to solve explicitly a dual problem.

(ii) In the special case when $m=1$ and $D_{1}$ is as in (1.3), the primal problem (1.1) reduces to (we drop the subscript ' 1 ' for brevity)

$$
\text { find } \bar{x} \in \mathcal{H} \text { such that } z \in A \bar{x}+L^{*}(B(L \bar{x}-r))+C \bar{x},
$$

the dual problem (1.2) reduces to

$$
\text { find } \bar{v} \in \mathcal{G} \text { such that } \quad-r \in-L\left((A+C)^{-1}\left(z-L^{*} \bar{v}\right)\right)+B^{-1} \bar{v},
$$

and the algorithm is governed by the iteration

$$
\mid \begin{aligned}
& y_{1, n}=x_{n}-\gamma_{n}\left(C x_{n}+L^{*} v_{n}+a_{1, n}\right) \\
& y_{2, n}=v_{n}+\gamma_{n}\left(L x_{n}+a_{2, n}\right) \\
& p_{1, n}=J_{\gamma_{n} A}\left(y_{1, n}+\gamma_{n} z\right)+b_{1, n} \\
& p_{2, n}=J_{\gamma_{n} B^{-1}}\left(y_{2, n}-\gamma_{n} r\right)+b_{2, n} \\
& q_{1, n}=p_{1, n}-\gamma_{n}\left(C p_{1, n}+L^{*} p_{2, n}+c_{1, n}\right) \\
& q_{2, n}=p_{2, n}+\gamma_{n}\left(L p_{1, n}+c_{2, n}\right) \\
& x_{n+1}=x_{n}-y_{1, n}+q_{1, n} \\
& v_{n+1}=v_{n}-y_{2, n}+q_{2, n} .
\end{aligned}
$$

On the one hand, if $C: x \mapsto 0$, we recover the primal-dual setting of [10] and its algorithm ([10, Eq. (3.1)]). On the other hand, if $L: x \mapsto 0, B: y \mapsto\{0\}, z=0$, and $r=0$, (3.46) yields 
the problem studied in [45], and (3.48) without error terms and dual variables yields a primal algorithm proposed in that paper, namely

$$
\left\lfloor\begin{array}{l}
y_{1, n}=x_{n}-\gamma_{n} C x_{n} \\
p_{1, n}=J_{\gamma_{n}} y_{1, n} \\
q_{1, n}=p_{1, n}-\gamma_{n} C p_{1, n} \\
x_{n+1}=x_{n}-y_{1, n}+q_{1, n}
\end{array} .\right.
$$

Let us note that, even when we specialize (3.46) to $\mathcal{G}=\mathcal{H}$ and $L=\mathrm{Id}$, there does not appear to exist an alternative algorithm that splits $A, B$, and $C$ and uses explicit steps on the Lipschitzian operator $C$.

(iii) When $C: x \mapsto 0$ and, for every $i \in\{1, \ldots, m\}, D_{i}^{-1}: y \mapsto\{0\}$, we recover the primal-dual setting of [10, Theorem 3.8]. However, the algorithm we obtain is different from that proposed in that paper, and novel.

(iv) In general, the weak convergence results of Theorem 3.1(ii) cannot be improved to strong convergence without additional hypotheses on the operators such as those described in (ii)(e) and (ii)(f). Indeed, in the special case when (1.1) reduces to the problem of finding a zero of $A$, the primal component of (3.3) reduces to the proximal point algorithm, namely (set $C: x \mapsto 0$ in $(3.49))$

$$
(\forall n \in \mathbb{N}) \quad x_{n+1}=J_{\gamma_{n} A} x_{n}
$$

which is known to converge weakly but not strongly [7, 25].

\section{Minimization problems}

The proposed monotone operator splitting algorithm can be applied to a broader class of problems than that within the reach of existing splitting methods. It has therefore potential applications in the areas in which these methods have been used, e.g., partial differential equations [21, 30], mechanics [22, 31], variational inequalities [8, 18, 44], game theory [11], traffic theory [20], and evolution equations [3]. In this section, we focus on the application of the results of Section 3 to convex minimization problems.

Problem 4.1 Let $\mathcal{H}$ be a real Hilbert space, let $z \in \mathcal{H}$, let $m$ be a strictly positive integer, let $f \in \Gamma_{0}(\mathcal{H})$, and let $h: \mathcal{H} \rightarrow \mathbb{R}$ be convex and differentiable with a $\mu$-Lipschitzian gradient for some $\mu \in] 0,+\infty\left[\right.$. For every $i \in\{1, \ldots, m\}$, let $\mathcal{G}_{i}$ be a real Hilbert space, let $r_{i} \in \mathcal{G}_{i}$, let $g_{i} \in \Gamma_{0}\left(\mathcal{G}_{i}\right)$, let $\ell_{i} \in \Gamma_{0}\left(\mathcal{G}_{i}\right)$ be $1 / \nu_{i}$-strongly convex, for some $\left.\nu_{i} \in\right] 0,+\infty\left[\right.$, and suppose that $L_{i}: \mathcal{H} \rightarrow \mathcal{G}_{i}$ is a nonzero bounded linear operator. Consider the problem

$$
\underset{x \in \mathcal{H}}{\operatorname{minimize}} f(x)+\sum_{i=1}^{m}\left(g_{i} \square \ell_{i}\right)\left(L_{i} x-r_{i}\right)+h(x)-\langle x \mid z\rangle,
$$

and the dual problem

$$
\underset{v_{1} \in \mathcal{G}_{1}, \ldots, v_{m} \in \mathcal{G}_{m}}{\operatorname{minimize}}\left(f^{*} \square h^{*}\right)\left(z-\sum_{i=1}^{m} L_{i}^{*} v_{i}\right)+\sum_{i=1}^{m}\left(g_{i}^{*}\left(v_{i}\right)+\ell_{i}^{*}\left(v_{i}\right)+\left\langle v_{i} \mid r_{i}\right\rangle\right) .
$$


The following result is an offspring of Theorem 3.1.

Theorem 4.2 In Problem 4.1, suppose that

$$
z \in \operatorname{ran}\left(\partial f+\sum_{i=1}^{m} L_{i}^{*}\left(\partial g_{i} \square \partial \ell_{i}\right)\left(L_{i} \cdot-r_{i}\right)+\nabla h\right) .
$$

Let $\left(a_{1, n}\right)_{n \in \mathbb{N}},\left(b_{1, n}\right)_{n \in \mathbb{N}}$, and $\left(c_{1, n}\right)_{n \in \mathbb{N}}$ be absolutely summable sequences in $\mathcal{H}$ and, for every $i \in\{1, \ldots, m\}$, let $\left(a_{2, i, n}\right)_{n \in \mathbb{N}},\left(b_{2, i, n}\right)_{n \in \mathbb{N}}$, and $\left(c_{2, i, n}\right)_{n \in \mathbb{N}}$ be absolutely summable sequences in $\mathcal{G}_{i}$. Furthermore, set

$$
\beta=\max \left\{\mu, \nu_{1}, \ldots, \nu_{m}\right\}+\sqrt{\sum_{i=1}^{m}\left\|L_{i}\right\|^{2}},
$$

let $x_{0} \in \mathcal{H}$, let $\left(v_{1,0}, \ldots, v_{m, 0}\right) \in \mathcal{G}_{1} \oplus \cdots \oplus \mathcal{G}_{m}$, let $\left.\varepsilon \in\right] 0,1 /(\beta+1)\left[\right.$, let $\left(\gamma_{n}\right)_{n \in \mathbb{N}}$ be a sequence in $[\varepsilon,(1-\varepsilon) / \beta]$, and set

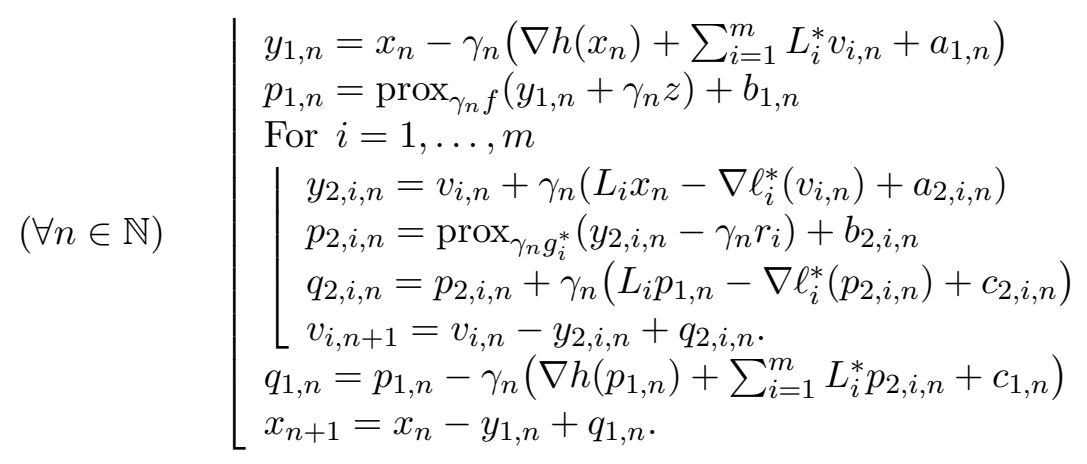

Then the following hold.

(i) $\sum_{n \in \mathbb{N}}\left\|x_{n}-p_{1, n}\right\|^{2}<+\infty$ and $(\forall i \in\{1, \ldots, m\}) \sum_{n \in \mathbb{N}}\left\|v_{i, n}-p_{2, i, n}\right\|^{2}<+\infty$.

(ii) There exist a solution $\bar{x}$ to (4.1) and a solution $\left(\overline{v_{1}}, \ldots, \overline{v_{m}}\right)$ to (4.2) such that the following hold.

(a) $z-\sum_{j=1}^{m} L_{j}^{*} \overline{v_{j}} \in \partial f(\bar{x})+\nabla h(\bar{x})$ and $(\forall i \in\{1, \ldots, m\}) L_{i} \bar{x}-r_{i} \in \partial g_{i}^{*}\left(\overline{v_{i}}\right)+\nabla \ell_{i}^{*}\left(\overline{v_{i}}\right)$.

(b) $x_{n} \rightarrow \bar{x}$ and $p_{1, n} \rightarrow \bar{x}$.

(c) $(\forall i \in\{1, \ldots, m\}) v_{i, n} \rightarrow \overline{v_{i}}$ and $p_{2, i, n} \rightarrow \overline{v_{i}}$.

(d) Suppose that $f$ or $h$ is uniformly convex at $\bar{x}$. Then $x_{n} \rightarrow \bar{x}$ and $p_{1, n} \rightarrow \bar{x}$.

(e) Suppose that, for some $i \in\{1, \ldots, m\}, g_{i}^{*}$ or $\ell_{i}^{*}$ is uniformly convex at $\overline{v_{i}}$. Then $v_{i, n} \rightarrow \overline{v_{i}}$ and $p_{2, i, n} \rightarrow \overline{v_{i}}$.

Proof. Let us first establish a connection between Problem 4.1 and Problem 1.1. To this end, let us define

$$
A=\partial f, C=\nabla h, \text { and } \quad(\forall i \in\{1, \ldots, m\}) \quad B_{i}=\partial g_{i} \text { and } D_{i}=\partial \ell_{i} .
$$

It is clear that (4.3) yields (3.1) and, using (2.7) and (2.8), that (4.5) yields (3.3). Moreover, it follows from [8, Theorem 20.40] that the operators $A$ and $\left(B_{i}\right)_{1 \leq i \leq m}$ are maximally monotone, and from [8, 
Proposition 17.10] that $C$ is monotone. On the other hand, for every $i \in\{1, \ldots, m\}$, it follows from the $1 / \nu_{i}$-strong convexity of $\ell_{i}$ and [8, Corollary 13.33 and Theorem 18.15] that $\ell_{i}^{*}$ is Fréchet differentiable on $\mathcal{G}_{i}$ with a $\nu_{i}$-Lipschitzian gradient, and from (2.7) that $D_{i}^{-1}=\nabla \ell_{i}^{*}$. Altogether, we can apply Theorem 3.1 to obtain the existence of a point $\bar{x} \in \mathcal{H}$ such that

$$
z \in \partial f(\bar{x})+\sum_{i=1}^{m} L_{i}^{*}\left(\left(\partial g_{i} \square \partial \ell_{i}\right)\left(L_{i} \bar{x}-r_{i}\right)\right)+\nabla h(\bar{x}),
$$

and of an $m$-tuple $\left(\overline{v_{1}}, \ldots, \overline{v_{m}}\right) \in \mathcal{G}_{1} \oplus \cdots \oplus \mathcal{G}_{m}$ such that

$$
(\exists x \in \mathcal{H}) \quad\left\{\begin{array}{l}
z-\sum_{j=1}^{m} L_{j}^{*} \overline{v_{j}} \in \partial f(x)+\nabla h(x) \\
(\forall i \in\{1, \ldots, m\}) \quad \overline{v_{i}} \in\left(\partial g_{i} \square \partial \ell_{i}\right)\left(L_{i} x-r_{i}\right),
\end{array}\right.
$$

that satisfy (i) and (ii). It remains to show that $\bar{x}$ solve $(4.1)$ and $\left(\overline{v_{1}}, \ldots, \overline{v_{m}}\right)$ solves $(4.2)$. We first observe that since, for every $i \in\{1, \ldots, m\}$, dom $\ell_{i}^{*}=\mathcal{G}_{i}[8$, Proposition 24.27] yields

$$
(\forall i \in\{1, \ldots, m\}) \quad \partial g_{i} \square \partial \ell_{i}=\partial\left(g_{i} \square \ell_{i}\right) .
$$

On the other hand, it follows from [8, Corollary 16.38(iii) and Proposition 17.26(i)] that

$$
\partial(f+h-\langle\cdot \mid z\rangle)=\partial f+\nabla h-z .
$$

As a result, we derive from (4.7) that

$$
0 \in \partial(f+h-\langle\cdot \mid z\rangle)(\bar{x})+\sum_{i=1}^{m} L_{i}^{*}\left(\partial\left(g_{i} \square \ell_{i}\right)\left(L_{i} \bar{x}-r_{i}\right)\right) .
$$

However, since (4.3) and [8, Proposition 16.5(ii)] imply that

$$
\partial(f+h-\langle\cdot \mid z\rangle)+\sum_{i=1}^{m} L_{i}^{*}\left(\partial\left(g_{i} \square \ell_{i}\right)\right)\left(L_{i} \cdot-r_{i}\right) \subset \partial\left(f+h-\langle\cdot \mid z\rangle+\sum_{i=1}^{m}\left(g_{i} \square \ell_{i}\right) \circ\left(L_{i} \cdot-r_{i}\right)\right),
$$

it follows from (4.11) that

$$
0 \in \partial\left(f+h-\langle\cdot \mid z\rangle+\sum_{i=1}^{m}\left(g_{i} \square \ell_{i}\right) \circ\left(L_{i} \cdot-r_{i}\right)\right)(\bar{x}) .
$$

Thus, Fermat's rule [8, Theorem 16.2] asserts that $\bar{x}$ solves (4.1). Finally, to show that $\left(\overline{v_{1}}, \ldots, \overline{v_{m}}\right)$ solves (4.2), we first note that it follows from (4.10), (2.7), and [8, Proposition 15.2] that

$$
(\partial f+\nabla h)^{-1}=(\partial(f+h))^{-1}=\partial(f+h)^{*}=\partial\left(f^{*} \square h^{*}\right) .
$$

Likewise, (4.9) and [8, Proposition 13.21(i)] yield

$$
(\forall i \in\{1, \ldots, m\}) \quad\left(\partial g_{i} \square \partial \ell_{i}\right)^{-1}=\partial\left(g_{i} \square \ell_{i}\right)^{*}=\partial\left(g_{i}^{*}+\ell_{i}^{*}\right) .
$$

Hence, combining (4.8), (4.14), and (4.15), we obtain

$$
(\exists x \in \mathcal{H}) \quad\left\{\begin{array}{l}
x \in \partial\left(f^{*} \square h^{*}\right)\left(z-\sum_{j=1}^{m} L_{j}^{*} \overline{j_{j}}\right) \\
(\forall i \in\{1, \ldots, m\}) \quad L_{i} x-r_{i} \in \partial\left(g_{i}^{*}+\ell_{i}^{*}\right)\left(\overline{v_{i}}\right)
\end{array}\right.
$$


and therefore

$$
(\exists x \in \mathcal{H})\left\{\begin{array}{l}
-\left(L_{i} x\right)_{1 \leq i \leq m} \in-\left(\underset{i=1}{\times} L_{i}\right)\left(\partial\left(f^{*} \square h^{*}\right)\left(z-\sum_{j=1}^{m} L_{j}^{*} \overline{v_{j}}\right)\right) \\
\left(L_{i} x\right)_{1 \leq i \leq m} \in \underset{i=1}{\times} \partial\left(g_{i}^{*}+\ell_{i}^{*}+\left\langle\cdot \mid r_{i}\right\rangle\right)\left(\overline{v_{i}}\right) .
\end{array}\right.
$$

Hence, using [8, Propositions 16.5(ii) and 16.8] and the notation (2.1),

$$
\begin{aligned}
(0, \ldots, 0) \in & -\left(\underset{i=1}{\times} L_{i}\right)\left(\partial\left(f^{*} \square h^{*}\right)\left(z-\sum_{j=1}^{m} L_{j}^{*} \overline{v_{j}}\right)\right)+\underset{i=1}{\times} \partial\left(g_{i}^{*}+\ell_{i}^{*}+\left\langle\cdot \mid r_{i}\right\rangle\right)\left(\overline{v_{i}}\right) \\
= & -\left(\bigoplus_{i=1}^{m} L_{i}^{*}\right)^{*}\left(\partial\left(f^{*} \square h^{*}\right)\left(z-\left(\bigoplus_{i=1}^{m} L_{i}^{*}\right)\left(\overline{v_{1}}, \ldots, \overline{v_{m}}\right)\right)\right) \\
& +\partial\left(\bigoplus_{i=1}^{m}\left(g_{i}^{*}+\ell_{i}^{*}+\left\langle\cdot \mid r_{i}\right\rangle\right)\right)\left(\overline{v_{1}}, \ldots, \overline{v_{m}}\right) \\
\subset & \partial\left(\left(f^{*} \square h^{*}\right)\left(z-\left(\bigoplus_{i=1}^{m} L_{i}^{*}\right) \cdot\right)+\bigoplus_{i=1}^{m}\left(g_{i}^{*}+\ell_{i}^{*}+\left\langle\cdot \mid r_{i}\right\rangle\right)\right)\left(\overline{v_{1}}, \ldots, \overline{v_{m}}\right) .
\end{aligned}
$$

In other words, by Fermat's rule, $\left(\overline{v_{1}}, \ldots, \overline{v_{m}}\right)$ solves (4.2). Finally, the strong convergence claims in (ii)(d) and (ii)(e) follow from Theorem 3.1(ii)(e)\&(ii)(f) since the uniform convexity of a function $\varphi \in \Gamma_{0}(\mathcal{H})$ at a point of the domain of $\partial \varphi$ implies the uniform monotonicity of $\partial \varphi$ at that point [46, Section 3.4]. प

In the following proposition we give conditions under which (4.3) is satisfied.

Proposition 4.3 Suppose that (4.1) has at least one solution and set

$$
S=\left\{\left(L_{i} x-y_{i}\right)_{1 \leq i \leq m} \mid x \in \operatorname{dom} f \text { and }(\forall i \in\{1, \ldots, m\}) y_{i} \in \operatorname{dom} g_{i}+\operatorname{dom} \ell_{i}\right\} .
$$

Then (4.3) is satisfied if one of the following holds.

(i) $\left(r_{1}, \ldots, r_{m}\right) \in \operatorname{sri} S$.

(ii) For every $i \in\{1, \ldots, m\}, g_{i}$ or $\ell_{i}$ is real-valued.

(iii) $\mathcal{H}$ and $\left(\mathcal{G}_{i}\right)_{1 \leq i \leq m}$ are finite-dimensional, and there exists $x \in \operatorname{ridom} f$ such that

$$
(\forall i \in\{1, \ldots, m\}) \quad L_{i} x-r_{i} \in \operatorname{ridom} g_{i}+\operatorname{ridom} \ell_{i} .
$$

Proof. It follows from (4.19) and [8, Proposition 12.6(ii)] that

$$
\begin{aligned}
S & =\left\{\left(L_{i} x-y_{i}\right)_{1 \leq i \leq m} \mid x \in \operatorname{dom} f \text { and }(\forall i \in\{1, \ldots, m\}) \quad y_{i} \in \operatorname{dom}\left(g_{i} \square \ell_{i}\right)\right\} \\
& =\left\{\left(L_{i} x-y_{i}\right)_{1 \leq i \leq m} \mid x \in \operatorname{dom}(f+h-\langle\cdot \mid z\rangle) \text { and }\left(y_{i}\right)_{1 \leq i \leq m} \in \underset{i=1}{\mathrm{X}} \operatorname{dom}\left(g_{i} \square \ell_{i}\right)\right\} \\
& =\left({\underset{i=1}{\mathrm{X}} L_{i}}_{i}\right)(\operatorname{dom}(f+h-\langle\cdot \mid z\rangle))-\operatorname{dom} \bigoplus_{i=1}^{m}\left(g_{i} \square \ell_{i}\right) .
\end{aligned}
$$


(i): In view of (4.21),

$$
\begin{aligned}
& \left(r_{1}, \ldots, r_{m}\right) \in \operatorname{sri} S \\
& \quad \Rightarrow \quad(0, \ldots, 0) \in \operatorname{sri}\left(\left({\underset{i=1}{X}}_{X}^{m}\right)(\operatorname{dom}(f+h-\langle\cdot \mid z\rangle))-\operatorname{dom} \bigoplus_{i=1}^{m}\left(g_{i} \square \ell_{i}\right)\left(\cdot-r_{i}\right)\right) .
\end{aligned}
$$

Hence, since $\left(X_{i=1}^{m} L_{i}\right)^{*}=\bigoplus_{i=1}^{m} L_{i}^{*}$, it follows from (4.9), (4.10), and [8, Theorem 16.37(i)] that

$$
\begin{aligned}
\partial f+\sum_{i=1}^{m} L_{i}^{*}\left(\partial g_{i} \square \partial \ell_{i}\right)\left(L_{i} \cdot-r_{i}\right)+\nabla h-z & =\partial(f+h-\langle\cdot \mid z\rangle)+\sum_{i=1}^{m} L_{i}^{*}\left(\partial\left(g_{i} \square \ell_{i}\right)\right)\left(L_{i} \cdot-r_{i}\right) \\
& =\partial\left(f+h-\langle\cdot \mid z\rangle+\sum_{i=1}^{m}\left(g_{i} \square \ell_{i}\right) \circ\left(L_{i} \cdot-r_{i}\right)\right) .
\end{aligned}
$$

Since (4.1) has at least one solution it follows from Fermat's rule that 0 is in the range of the right-hand side of (4.23), which shows that (4.3) holds.

(ii) $\Rightarrow$ (i): We have $(\forall i \in\{1, \ldots, m\}) \operatorname{dom} g_{i}+\operatorname{dom} \ell_{i}=\mathcal{G}_{i}$. Therefore (4.19) yields $S=\bigoplus_{i=1}^{m} \mathcal{G}_{i}$.

(iii) $\Rightarrow($ i): We have sri $S=\operatorname{ri} S$. However, it follows from (4.21) and [8, Corollary 6.15] that

$$
\begin{aligned}
& \operatorname{ri} S=\operatorname{ri}\left(\left(\underset{i=1}{X} L_{i}\right)(\operatorname{dom}(f+h-\langle\cdot \mid z\rangle))-\operatorname{dom} \bigoplus_{i=1}^{m}\left(g_{i} \square \ell_{i}\right)\right) \\
& =\operatorname{ri}\left(\underset{i=1}{X} L_{i}\right)(\operatorname{dom} f)-\operatorname{ridom} \bigoplus_{i=1}^{m}\left(g_{i} \square \ell_{i}\right) \\
& =\left(\underset{i=1}{\underset{X}{X}} L_{i}\right)(\operatorname{ridom} f)-\underset{i=1}{\underset{X}{X}} \operatorname{ridom}\left(g_{i} \square \ell_{i}\right) \\
& =\left(\underset{i=1}{\underset{X}{X}} L_{i}\right)(\operatorname{ridom} f)-\underset{i=1}{\times}\left(\operatorname{ridom} g_{i}+\operatorname{ridom} \ell_{i}\right) .
\end{aligned}
$$

Hence $\left(r_{1}, \ldots, r_{m}\right) \in \operatorname{sri} S \Leftrightarrow(\exists x \in \operatorname{ridom} f)(\forall i \in\{1, \ldots, m\}) L_{i} x-r_{i} \in \operatorname{ridom} g_{i}+\operatorname{ridom} \ell_{i}$.

Remark 4.4 In Problem 4.1, if each function $\ell_{i}$ is the indicator function of $\{0\}$, then (4.1) reduces to

$$
\underset{x \in \mathcal{H}}{\operatorname{minimize}} f(x)+\sum_{i=1}^{m} g_{i}\left(L_{i} x-r_{i}\right)+h(x)-\langle x \mid z\rangle .
$$

Even in this special case, the algorithm resulting from (4.5) is new. This observation remains valid if we further assume that $h: x \mapsto 0$.

\section{References}

[1] F. Acker and M. A. Prestel, Convergence d'un schéma de minimisation alternée, Ann. Fac. Sci. Toulouse V. Sér. Math., vol. 2, pp. 1-9, 1980. 
[2] G. Alduncin, Composition duality principles for mixed variational inequalities, Math. Comput. Modelling, vol. 41, pp. 639-654, 2005.

[3] H. Attouch, L. M. Briceño-Arias, and P. L. Combettes, A parallel splitting method for coupled monotone inclusions, SIAM J. Control Optim., vol. 48, pp. 3246-3270, 2010.

[4] H. Attouch, P. Redont, and A. Soubeyran, A new class of alternating proximal minimization algorithms with costs-to-move, SIAM J. Optim., vol. 18, pp. 1061-1081, 2007.

[5] H. Attouch and M. Théra, A general duality principle for the sum of two operators, J. Convex Anal., vol. 3, pp. 1-24, 1996.

[6] J.-F. Aujol and A. Chambolle, Dual norms and image decomposition models, Int. J. Comput. Vision, vol. 63, pp. 85-104, 2005.

[7] H. H. Bauschke, J. V. Burke, F. R. Deutsch, H. S. Hundal, and J. D. Vanderwerff, A new proximal point iteration that converges weakly but not in norm, Proc. Amer. Math. Soc., vol. 133, pp. 1829-1835, 2005.

[8] H. H. Bauschke and P. L. Combettes, Convex Analysis and Monotone Operator Theory in Hilbert Spaces, Springer, New York, 2011.

[9] H. H. Bauschke, P. L. Combettes, and S. Reich, The asymptotic behavior of the composition of two resolvents, Nonlinear Anal., vol. 60, pp. 283-301, 2005.

[10] L. M. Briceño-Arias and P. L. Combettes, A monotone+skew splitting model for composite monotone inclusions in duality, SIAM J. Optim., to appear.

[11] L. M. Briceño-Arias and P. L. Combettes, Monotone operator methods for Nash equilibria in non-potential games. http://arxiv.org/abs/1106.0144

[12] A. Chambolle and P.-L. Lions, Image recovery via total variation minimization and related problems, Numer. Math., vol. 76, pp. 167-188, 1997.

[13] P. L. Combettes, Solving monotone inclusions via compositions of nonexpansive averaged operators, Optimization, vol. 53, pp. 475-504, 2004.

[14] P. L. Combettes, Dinh Dũng, and B. C. Vũ, Proximity for sums of composite functions, J. Math. Anal. Appl., vol. 380, pp. 680-688, 2011.

[15] P. L. Combettes and V. R. Wajs, Signal recovery by proximal forward-backward splitting, Multiscale Model. Simul., vol. 4, pp. 1168-1200, 2005.

[16] J. Eckstein and M. C. Ferris, Smooth methods of multipliers for complementarity problems, Math. Programming, vol. 86, pp. 65-90, 1999.

[17] I. Ekeland and R. Temam, Analyse Convexe et Problèmes Variationnels, Dunod, Paris, 1974; Convex Analysis and Variational Problems, SIAM, Philadelphia, PA, 1999.

[18] F. Facchinei and J.-S. Pang, Finite-Dimensional Variational Inequalities and Complementarity Problems, Springer-Verlag, New York, 2003.

[19] W. Fenchel, Convex Cones, Sets and Functions, Princeton University, Princeton, NJ, 1953.

[20] M. Fukushima, The primal Douglas-Rachford splitting algorithm for a class of monotone mappings with applications to the traffic equilibrium problem, Math. Programming, vol. 72, pp. 1-15, 1996.

[21] D. Gabay, Applications of the method of multipliers to variational inequalities, in: M. Fortin and R. Glowinski (eds.), Augmented Lagrangian Methods: Applications to the Numerical Solution of Boundary Value Problems, pp. 299-331. North-Holland, Amsterdam, 1983.

[22] R. Glowinski and P. Le Tallec (eds.), Augmented Lagrangian and Operator-Splitting Methods in Nonlinear Mechanics, SIAM, Philadelphia, 1989. 
[23] C. J. Goh and X. Q. Yang, Duality in Optimization and Variational Inequalities, Taylor \& Francis, London, 2002.

[24] E. G. Gol'shteı̆n, Teoriya Dvoistvennosti v Matematicheskom Programmirovanii i ee Prilozheniya (Duality Theory in Mathematical Programming and Its Applications), Nauka, Moscow, 1971.

[25] O. Güler, On the convergence of the proximal point algorithm for convex minimization. SIAM J. Control Optim., vol. 29, pp. 403-419, 1991.

[26] I. V. Konnov, The splitting method with a linear search for direct-dual variational inequalities, Comput. Math. Math. Phys., vol. 43, pp. 494-507, 2003.

[27] V. N. Lebedev and N. T. Tynjanskiı̌, Duality theory of concave-convex games, Soviet Math. Dokl., vol. 8, pp. 752-756, 1967.

[28] J. Luque, Convolutions of Maximal Monotone Mappings, Technical report LIDS-P-1597, MIT Libraries, Cambridge, MA, 1986. http://mit.dspace.org/handle/1721.1/2953

[29] L. McLinden, An extension of Fenchel's duality theorem to saddle functions and dual minimax problems, Pacific J. Math., vol. 50, pp. 135-158, 1974.

[30] B. Mercier, Topics in Finite Element Solution of Elliptic Problems (Lectures on Mathematics, no. 63). Tata Institute of Fundamental Research, Bombay, 1979.

[31] B. Mercier, Inéquations Variationnelles de la Mécanique (Publications Mathématiques d'Orsay, no. 80.01). Université de Paris-XI, Orsay, France, 1980.

[32] J.-J. Moreau, Théorèmes "inf-sup," C. R. Acad. Sci. Paris Sér. A Math., vol. 258, pp. 2720-2722, 1964.

[33] J.-J. Moreau, Fonctionnelles Convexes, Séminaire Jean Leray sur les Équations aux Dérivées Partielles, no. 2, Collège de France, Paris, 1966-1967. Corrected reprint: Edizioni del Dipartimento di Ingegneria Civile, Università di Roma Tor Vergata, Rome, 2003.

[34] U. Mosco, Dual variational inequalities, J. Math. Anal. Appl., vol. 40, pp. 202-206, 1972.

[35] A. Moudafi, On the stability of the parallel sum of maximal monotone operators, J. Math. Anal. Appl., vol. 199 , pp. $478-488,1996$.

[36] G. B. Passty, The parallel sum of nonlinear monotone operators, Nonlinear Anal., vol. 10, pp. 215-227, 1986.

[37] T. Pennanen, Dualization of generalized equations of maximal monotone type, SIAM J. Optim., vol. 10, pp. 809-835, 2000.

[38] S. M. Robinson, Composition duality and maximal monotonicity, Math. Programming, vol. 85, pp. 1-13, 1999.

[39] R. T. Rockafellar, Minimax theorems and conjugate saddle-functions, Math. Scand., vol. 14, pp. 151-173, 1964.

[40] R. T. Rockafellar, Duality and stability in extremum problems involving convex functions, Pacific J. Math., vol. 21, pp. 167-187, 1967.

[41] R. T. Rockafellar, Conjugate Duality and Optimization. SIAM, Philadelphia, PA, 1974.

[42] R. T. Rockafellar, Monotone operators and the proximal point algorithm, SIAM J. Control Optim., vol. 14, pp. 877-898, 1976.

[43] A. Seeger, Direct and inverse addition in convex analysis and applications, J. Math. Anal. Appl., vol. 148, pp. 317-349, 1990.

[44] P. Tseng, Applications of a splitting algorithm to decomposition in convex programming and variational inequalities, SIAM J. Control Optim., vol. 29, pp. 119-138, 1991. 
[45] P. Tseng, A modified forward-backward splitting method for maximal monotone mappings, SIAM J. Control Optim., vol. 38, pp. 431-446, 2000.

[46] C. Zălinescu, Convex Analysis in General Vector Spaces, World Scientific, River Edge, NJ, 2002. 